\title{
Relationship between Uric Acid Level and Severity of Acute Primary Cerebral Infarction: A Cross-Sectional Study
}

\author{
Ruying Wang $\mathbb{D}^{1}{ }^{1}$ Yi Zhong, ${ }^{2}$ Quan Zhou, ${ }^{3}$ and Ping Xu $\mathbb{D}^{2}$ \\ ${ }^{1}$ Nanhua University, Hengyang, Hunan, China \\ ${ }^{2}$ Department of Neurology, The First People's Hospital of Changde City, Changde, Hunan, China \\ ${ }^{3}$ Ministry of Science and Education, The First People's Hospital of Changde City, Changde, Hunan, China
}

Correspondence should be addressed to Ping Xu; 1301154724@qq.com

Received 2 January 2020; Revised 29 April 2020; Accepted 26 May 2020; Published 23 June 2020

Academic Editor: Vida Demarin

Copyright (c) 2020 Ruying Wang et al. This is an open access article distributed under the Creative Commons Attribution License, which permits unrestricted use, distribution, and reproduction in any medium, provided the original work is properly cited.

\begin{abstract}
Numerous studies have shown that uric acid (UA) is associated with cerebrovascular disease, but whether UA is a protective factor or worsens the risk of developing cerebrovascular disease remains controversial. This study investigated the relationship between UA levels detected at admission and the severity of acute primary cerebral infarction. This cross-sectional study enrolled patients with acute primary cerebral infarction $(N=238,157 \mathrm{men})$. We designated the levels of serum UA measured at the time of admission as the independent variable and the degree of neurological impairment at admission as the dependent variable. The National Institutes of Health Stroke Scale (NIHSS) was used to assess the extent of neurological dysfunction: NIHSS $\leq 5$, minor stroke; NIHSS $>5$, moderate to severe stroke. There was a statistically significant difference in UA levels between patients with mild cerebral infarctions (NIHSS $\leq 5)$ and those with moderate or severe cerebral infarctions $($ NIHSS $>5)(P<0.0001)$. After adjusting for confounding factors, the serum UA level was found to be nonlinearly related to NIHSS, and the inflection point was $372 \mu \mathrm{mol} / \mathrm{L}$. The extent of the influence and confidence interval was $0.99(0.98,0.99)$ on the left side of the inflection point and $1.00(1.00,1.01)$ on the right side. There was a nonlinear relationship between the UA level measured on admission and the degree of neurological impairment in patients with acute primary cerebral infarction. When UA was $<372 \mu \mathrm{mol} / \mathrm{L}$, it was negatively correlated with the degree of neurological impairment in patients with acute cerebral infarction, but when UA was $\geq 372 \mu \mathrm{mol} / \mathrm{L}$, the protective effect of UA disappeared.
\end{abstract}

\section{Introduction}

With its highest incidences in Asia-especially in China-and Eastern Europe, cerebral infarction remains a global concern. Moreover, according to the European Stroke Burden Report, no changes in the current incidence of cerebral infarctions will increase the total number of stroke events by more than $30 \%$ between 2015 and 2035 [1,2]. However, the mechanism underlying the onset of cerebral infarction remains to be elucidated. Most studies of cerebral infarction have focused on its treatment and prognosis [3]. Some studies suggest that the neuroendocrine status is related to the prognosis of patients with acute cerebral infarction [4]. Patients with severe neuroendocrine disorders have a poor prognosis. Studies have shown that serum uric acid (UA), a metabolite of purine [5], can predict cardiovascular and cerebrovascular accidents and is associated with early changes in neurological function. A meta-analysis found that high serum UA levels had a neuroprotective effect on the prognosis of patients with acute ischemic stroke [6]. However, the relationship between UA and cerebral infarction is still controversial. While some research has shown that UA is involved in the formation of cerebral atherosclerosis, is a risk factor for cerebral infarction, and can cause cardiovascular and cerebrovascular accidents $[7,8]$, a study has shown that serum UA has no relationship to cerebral infarction [9]. To help resolve this controversy, the present study investigated whether UA levels at admission were independently associated with the severity of acute primary cerebral infarction. 
TABLE 1: Relationship between UA and NIHSS in different models.

\begin{tabular}{|c|c|c|c|c|c|c|}
\hline \multirow{2}{*}{ Variable } & \multicolumn{2}{|c|}{ Crude model } & \multicolumn{2}{|c|}{ Model I } & \multicolumn{2}{|c|}{ Model II } \\
\hline & OR (95\% CI) & $P$ value & OR (95\% CI) & $P$ value & OR (95\% CI) & $P$ value \\
\hline $\mathrm{UA}(\mu \mathrm{mol} / \mathrm{L})$ & $0.99(0.99,0.99)$ & $<0.0001$ & $0.99(0.99,1.00)$ & $<0.0001$ & $0.99(0.99,0.99)$ & $<0.0001$ \\
\hline $\mathrm{UA}(10 \mu \mathrm{mol} / \mathrm{L})$ & $0.92(0.88,0.95)$ & $<0.0001$ & $0.92(0.89,0.95)$ & $<0.0001$ & $0.91(0.87,0.95)$ & $<0.0001$ \\
\hline \multicolumn{7}{|l|}{ UA (quartile) } \\
\hline Q1 & Reference & & Reference & & Reference & \\
\hline Q2 & $0.44(0.21,0.94)$ & 0.0337 & $0.46(0.21,0.98)$ & 0.0452 & $0.44(0.18,1.04)$ & 0.0602 \\
\hline Q3 & $0.13(0.05,0.34)$ & $<0.0001$ & $0.14(0.05,0.35)$ & $<0.0001$ & $0.13(0.04,0.37)$ & 0.0002 \\
\hline Q4 & $0.15(0.06,0.37)$ & $<0.0001$ & $0.17(0.07,0.42)$ & 0.0001 & $0.14(0.05,0.42)$ & 0.0004 \\
\hline$P$ for trend & & $<0.0001$ & & $<0.0001$ & & $<0.0001$ \\
\hline
\end{tabular}

Abbreviations: CI: confidence interval. Model I adjusted for sex and age; Model II adjusted for sex, age, systolic BP, diastolic BP, hypertension, diabetes, coronary artery disease, smoking, drinking, total cholesterol, triglyceride, HDL cholesterol, LDL cholesterol, homocysteine, creatinine, and urea nitrogen.

\section{Materials and Methods}

2.1. Research Design. This cross-sectional study explored the relationship between UA and the severity of acute primary cerebral infarction. The level of serum UA at the time of admission was selected as the independent variable and the degree of neurological impairment at admission as the dependent variable.

2.2. Research Population. We collected baseline data from patients who were hospitalized in the Department of Neurology, the First People's Hospital of Changde City, from November 1, 2018, to August 31, 2019. The criteria for inclusion in this study were as follows: (1) incidence time $<48$ hours and (2) an acute cerebral infarction that was confirmed by MRI during hospitalization. Exclusion criteria were as follows: (1) a history of cerebral infarction, malignant tumor, gouty arthritis, kidney disease, systemic infection, or an autoimmune disease; (2) the recent intake of drugs related to UA metabolism; and (3) missing data. All data were obtained from the participating hospital's electronic medical record system.

2.3. Method for Measuring the Degree of Neurological Dysfunction. The National Institutes of Health Stroke Scale (NIHSS) was used to assess the extent of neurological dysfunction. The NIHSS value was assessed and recorded by a neurologist at the time of admission. An NIHSS score of $\leq 5$ was considered to indicate a minor stroke; NIHSS scores of $>5$, moderate to severe stroke [10-12].

2.4. Measurement of UA. The specific process of UA measurement was as follows: (1) Venous blood was collected on the first day of the patient's admission and sent to the laboratory through our hospital's transportation system. (2) UA activity was measured using an oxidase method UA kit, the performance of which was approved by the national testing agency.

2.5. Statistical Analysis. We represent continuous variables in two ways. The continuous distribution of normal variables is expressed as the mean \pm standard deviation, while skewed distributions are expressed as medians and interquartile ranges (Q1-Q3). Classification variables are expressed in terms of frequency or percentage. We used a one-way analysis of variance (normal distribution), the Kruskal-Wallis H (skewed distribution) test, and the chisquare test (categorical variables) to examine differences between different UA groups. Our method of data analysis can be divided into two steps. In the first step, we explored the linear relationship between UA and NIHSS by using three linear regression models: Model 1, without covariate adjustments; Model 2, adjusted for social demographic data only, including age and sex; and Model 3, adjusted for all the covariates shown in Table 1. In the second step, we explored the nonlinear relationship between UA and NIHSS and performed a smooth curve fit. If nonlinearity was detected, we first calculated the inflection point using a recursive algorithm and then constructed a two-stage linear regression on both sides. The optimal fit model was determined based on the log likelihood ratio test value. To ensure the robustness of the data analysis, we conducted a sensitivity analysis. We converted the UA into a categorical variable and calculated the $P$ value of the trend. The purpose of the test was to verify the results of treating UA as a continuous variable and to determine the possibility of nonlinearity. All the analyses were performed with the statistical software package R (http://www.R-project.org, the R Foundation) and EmpowerStats (http://www.empowerstats.com, X\&Y Solutions, Inc., Boston, MA). $P$ values of $<0.05$ (both sides) were considered statistically significant.

\section{Results}

3.1. Participant Selection. Based on our strict screening criteria, a total of 238 patients were selected for final data analysis. A total of 51 were excluded, including 10 patients with a history of kidney disease, two patients with a history of cancer, and 39 patients with an onset time of more than 48 hours (see Figure 1 for a flow chart).

3.2. Baseline Characteristics of Participants. The baseline data characteristics of the patients are shown in Table 2 . The average age of the 238 subjects was $64.41 \pm 10.76$ years, $65.97 \%$ of whom were men. There was statistically significant difference in triglyceride, creatinine, homocysteine, sex, smoking, and NIHSS across quartiles of UA (all $P<0.05$ ), but no difference 


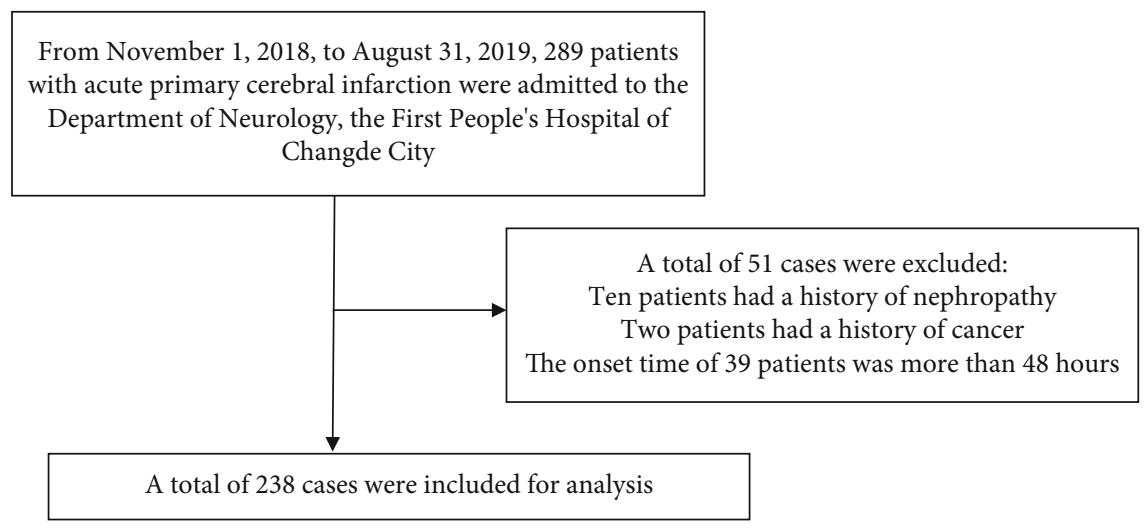

FIGURE 1: A flow chart.

TABLE 2: Baseline characteristics of the participants $(N=238)$.

\begin{tabular}{|c|c|c|c|c|c|}
\hline \multirow{2}{*}{ Characteristic } & \multicolumn{4}{|c|}{ UA quartiles $(\mu \mathrm{mol} / \mathrm{L})$} & \multirow[t]{2}{*}{$P$ value } \\
\hline & Q1 (103-256) & Q2 (262-322) & Q3 (323-384) & Q4 (387-573) & \\
\hline No. of participants & 58 & 59 & 57 & 64 & \\
\hline Age (years, median, Q1-Q3) & $67.50(56.25-73.75)$ & $64.00(55.00-74.00)$ & $65.00(57.00-71.00)$ & $62.00(53.00-69.00)$ & 0.182 \\
\hline Systolic BP $(\mathrm{mmHg}$, mean $\pm \mathrm{sd})$ & $160.71 \pm 25.80$ & $155.22 \pm 22.96$ & $155.47 \pm 20.47$ & $161.81 \pm 23.60$ & 0.271 \\
\hline Diastolic BP (mmHg, median, Q1-Q3) & $86.00(78.25-98.75)$ & $85.00(78.00-95.50)$ & $84.00(78.00-94.00)$ & $88.00(79.00-100.25)$ & 0.266 \\
\hline Total cholesterol (mmol/L, median, Q1-Q3) & $4.50(3.87-5.00)$ & $4.64(4.21-5.37)$ & $4.48(4.20-4.93)$ & $4.80(4.08-5.40)$ & 0.521 \\
\hline Triglyceride (mmol/L, median, Q1-Q3) & $1.39(0.98-1.99)$ & $1.44(1.11-1.97)$ & $1.80(1.21-2.45)$ & $1.67(1.36-2.76)$ & 0.009 \\
\hline HDL cholesterol (mmol/L, median, Q1-Q3) & $1.12(0.93-1.31)$ & $1.09(0.94-1.28)$ & $1.02(0.91-1.15)$ & $1.03(0.95-1.17)$ & 0.107 \\
\hline LDL cholesterol (mmol/L, median, Q1-Q3) & $2.51(2.09-3.20)$ & $2.60(2.25-3.40)$ & $2.64(2.39-2.98)$ & $2.70(2.25-3.21)$ & 0.772 \\
\hline Creatinine $(\mu \mathrm{mol} / \mathrm{L}$, median, Q1-Q3) & $66.00(54.00-75.00)$ & $69.00(60.00-85.00)$ & $76.00(67.00-88.00)$ & $87.50(70.00-104.00)$ & $<0.001$ \\
\hline Urea nitrogen $(\mu \mathrm{mol} / \mathrm{L}$, median, Q1-Q3) & $5.42(4.56-6.46)$ & $5.45(4.59-6.32)$ & $5.51(4.41-6.45)$ & $5.33(4.39-6.37)$ & 0.944 \\
\hline Homocysteine ( $\mu \mathrm{mol} / \mathrm{L}$, median, Q1-Q3) & $11.64(8.90-14.28)$ & $12.00(9.87-14.61)$ & $12.91(10.40-15.30)$ & $13.05(10.87-16.91)$ & 0.015 \\
\hline $\operatorname{Sex}(n, \%)$ & & & & & $<0.001$ \\
\hline Male & $27(46.55 \%)$ & $38(64.41 \%)$ & $39(68.42 \%)$ & $53(82.81 \%)$ & \\
\hline Female & $31(53.45 \%)$ & $21(35.59 \%)$ & $18(31.58 \%)$ & $11(17.19 \%)$ & \\
\hline Hypertension $(n, \%)$ & & & & & 0.337 \\
\hline No & $25(43.10 \%)$ & $24(40.68 \%)$ & $16(28.07 \%)$ & $26(40.62 \%)$ & \\
\hline Yes & $33(56.90 \%)$ & $35(59.32 \%)$ & $41(71.93 \%)$ & $38(59.38 \%)$ & \\
\hline Diabetes $(n, \%)$ & & & & & 0.994 \\
\hline No & $49(84.48 \%)$ & $50(84.75 \%)$ & $48(84.21 \%)$ & $55(85.94 \%)$ & \\
\hline Yes & $9(15.52 \%)$ & $9(15.25 \%)$ & $9(15.79 \%)$ & $9(14.06 \%)$ & \\
\hline Coronary artery disease $(n, \%)$ & & & & & 0.096 \\
\hline No & $46(79.31 \%)$ & $54(91.53 \%)$ & $53(92.98 \%)$ & $57(89.06 \%)$ & \\
\hline Yes & $12(20.69 \%)$ & $5(8.47 \%)$ & $4(7.02 \%)$ & $7(10.94 \%)$ & \\
\hline Smoking $(n, \%)$ & & & & & 0.013 \\
\hline No & $45(77.59 \%)$ & $44(74.58 \%)$ & $35(61.40 \%)$ & $34(53.12 \%)$ & \\
\hline Yes & $13(22.41 \%)$ & $15(25.42 \%)$ & $22(38.60 \%)$ & $30(46.88 \%)$ & \\
\hline Drinking $(n, \%)$ & & & & & 0.111 \\
\hline No & $53(91.38 \%)$ & $44(74.58 \%)$ & $46(80.70 \%)$ & $50(78.12 \%)$ & \\
\hline Yes & $5(8.62 \%)$ & $15(25.42 \%)$ & $11(19.30 \%)$ & $14(21.88 \%)$ & \\
\hline NIHSS & & & & & $<0.001$ \\
\hline Minor stroke $(\mathrm{NIHSS} \leq 5)$ & $28(48.28 \%)$ & $40(67.80 \%)$ & $50(87.72 \%)$ & $55(85.94 \%)$ & \\
\hline Moderate to severe stroke (NIHSS $>5$ ) & $30(51.72 \%)$ & $19(32.20 \%)$ & $7(12.28 \%)$ & $9(14.06 \%)$ & \\
\hline
\end{tabular}

Note: the continuous variable of nonnormal distribution is calculated by Kruskal-Wallis H test. BP: blood pressure; HDL: high-density lipoprotein; LDL: lowdensity lipoprotein; UA: uric acid; NIHSS: National Institutes of Health Stroke Scale. 
TABLE 3: Univariate analysis for NIHSS.

\begin{tabular}{|c|c|c|c|}
\hline Covariate & Statistics & OR $(95 \% \mathrm{CI})$ & $P$ value \\
\hline \multicolumn{4}{|l|}{$\operatorname{Sex}(n, \%)$} \\
\hline Male & $157(65.97 \%)$ & Ref & \\
\hline Female & $81(34.03 \%)$ & $1.71(0.95,3.08)$ & 0.0726 \\
\hline Age (years) & $64.41 \pm 10.75$ & $1.04(1.01,1.07)$ & 0.0064 \\
\hline Systolic BP (mmHg) & $158.39 \pm 23.35$ & $0.99(0.98,1.00)$ & 0.2146 \\
\hline Diastolic BP (mmHg) & $87.84 \pm 15.03$ & $0.99(0.97,1.01)$ & 0.2367 \\
\hline \multicolumn{4}{|l|}{ Hypertension $(n, \%)$} \\
\hline No & $91(38.24 \%)$ & Ref & \\
\hline Yes & $147(61.76 \%)$ & $0.76(0.42,1.35)$ & 0.3468 \\
\hline \multicolumn{4}{|l|}{ Diabetes $(n, \%)$} \\
\hline No & $202(84.87 \%)$ & Ref & \\
\hline Yes & $36(15.13 \%)$ & $1.88(0.90,3.95)$ & 0.0940 \\
\hline \multicolumn{4}{|l|}{ Coronary artery disease $(n, \%)$} \\
\hline No & $210(88.24 \%)$ & Ref & \\
\hline Yes & $28(11.76 \%)$ & $5.22(2.29,11.89)$ & $<0.0001$ \\
\hline \multicolumn{4}{|l|}{ Smoking $(n, \%)$} \\
\hline No & $158(66.39 \%)$ & Ref & \\
\hline Yes & $80(33.61 \%)$ & $0.62(0.33,1.17)$ & 0.1373 \\
\hline \multicolumn{4}{|l|}{ Drinking $(n, \%)$} \\
\hline No & $193(81.09 \%)$ & Ref & \\
\hline Yes & $45(18.91 \%)$ & $0.83(0.39,1.76)$ & 0.6321 \\
\hline Total cholesterol (mmol/L) & $4.70 \pm 1.02$ & $0.89(0.67,1.18)$ & 0.4218 \\
\hline Triglyceride $(\mathrm{mmol} / \mathrm{L})$ & $1.94 \pm 1.25$ & $0.72(0.53,0.96)$ & 0.0528 \\
\hline HDL cholesterol (mmol/L) & $1.10 \pm 0.25$ & $2.09(0.68,6.48)$ & 0.2002 \\
\hline LDL cholesterol (mmol/L) & $2.74 \pm 0.80$ & $0.91(0.63,1.32)$ & 0.6152 \\
\hline Homocysteine ( $\mu \mathrm{mol} / \mathrm{L})$ & $13.90 \pm 7.82$ & $0.97(0.92,1.02)$ & 0.2616 \\
\hline Creatinine $(\mu \mathrm{mol} / \mathrm{L})$ & $78.90 \pm 28.88$ & $1.01(1.00,1.01)$ & 0.2593 \\
\hline Urea nitrogen $(\mu \mathrm{mol} / \mathrm{L})$ & $5.64 \pm 1.94$ & $1.23(1.06,1.43)$ & 0.0079 \\
\hline $\mathrm{UA}(\mu \mathrm{mol} / \mathrm{L})$ & $328.87 \pm 98.78$ & $0.99(0.99,0.99)$ & $<0.0001$ \\
\hline
\end{tabular}

Abbreviations: CI: confidence interval; OR: odds ratio.

in age, systolic blood pressure, diastolic blood pressure, total cholesterol, LDL cholesterol, HDL cholesterol, urea nitrogen, history of hypertension, history of coronary heart disease, history of diabetes, and drinking (all $P>0.05$ ).

3.3. Univariate Analysis of the Degree of Neurological Dysfunction. The results of the univariate analysis are listed in Table 3. The univariate analysis showed that sex; systolic blood pressure; diastolic blood pressure; histories of hypertension, diabetes, smoking, and drinking; total cholesterol; triglyceride; high-density lipoprotein; low-density lipoprotein; homocysteine; and creatinine were not associated with NIHSS. Age, urea nitrogen, and history of coronary heart disease were positively correlated with NIHSS, while UA negatively correlated with NIHSS.

3.4. The Relationship between UA and NIHSS according to Three Linear Regression Models. The results obtained from the adjusted and unadjusted models are presented in Table 1, which reveals that UA raw data and UA increased by $10 \mu \mathrm{mol} / \mathrm{L}$. The finding can be explained as follows: the use of the unadjusted model (crude model) indicated an $8 \%$ reduction in the risk of moderate to severe cerebral infarction (OR 0.92 , 95\% CI 0.88 to $0.95, P<0.0001$ ) for every $10 \mu \mathrm{mol} / \mathrm{L}$ increase in UA. In the minimal adjustment model (Model I), the results were interpreted as an $8 \%$ reduction in the risk of moderate to severe cerebral infarction (OR 0.92, 95\% CI 0.89 to $0.95, P<0.0001$ ) for every $10 \mu \mathrm{mol} / \mathrm{L}$ increase in UA. Finally, after we completely adjusted the variables (Model II), the results could be explained by a $9 \%$ increase in the risk of moderate to severe cerebral infarction for each UA increase of $10 \mu \mathrm{mol} / \mathrm{L}$ (OR $0.91,95 \%$ CI 0.87 to 0.95 , $P<0.0001)$. For the sensitivity analysis, we also treated UA as a quarantine (quartile) and observed the same trend. As UA concentration increased, the risk of moderate to severe cerebral infarction decreased. 


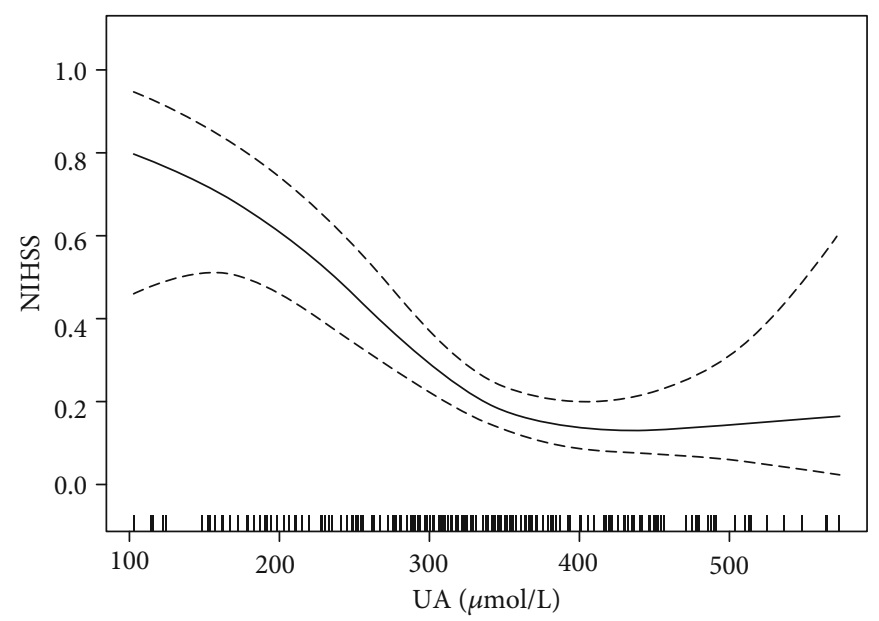

FIGURE 2: Relationship between UA ( $\mu \mathrm{mol} / \mathrm{L})$ and NIHSS. The middle line represents a smooth curve fit between the variables. The curves on both sides represent the $95 \%$ confidence interval for the fit. The model was adjusted according to sex, age, systolic BP, diastolic BP, hypertension, diabetes, coronary artery disease, smoking, drinking, total cholesterol, triglyceride, HDL cholesterol, LDL cholesterol, homocysteine, creatinine, and urea nitrogen.

3.5. The Analyses of Nonlinear Relationships. This study found a nonlinear relationship between UA and NIHSS (adjusted sex, age, systolic blood pressure, diastolic blood pressure, hypertension, diabetes, coronary artery disease, smoking, alcohol consumption, total cholesterol, triglycerides, high-density lipoprotein cholesterol, LDL cholesterol, homocysteine, creatinine, and urea nitrogen; Figure 2). Through the two-segment linear regression model, the inflection point was calculated to be $372 \mu \mathrm{mol} / \mathrm{L}$, and the influence and confidence intervals of the left and right sides of the inflection point were determined to be $0.99(0.98,0.99)$ and $1.00(1.00,1.01)$ (Table 4$)$, respectively.

\section{Discussion}

This study suggested that when UA was $<372 \mu \mathrm{mol} / \mathrm{L}$, UA was negatively correlated with the degree of neurological damage in patients with acute cerebral infarction. Yang et al. [13] found UA concentration to be negatively correlated with NIHSS scores. Two animal experiments found that treatment with UA after cerebral ischemia could maintain a high perfusion state and reduce nerve damage [14, 15]. Chiquete et al. [16] posited that the correlation between low concentration of UA and severity of acute cerebral infarction could be used as a marker of the degree of cerebral infarction. According to Arevalo-Lorido et al. [17], NIHSS scores decrease with increasing UA, even in patients with cerebral infarction and abnormal renal function. In performing a randomized, double-blind, placebocontrolled phase $2 \mathrm{~b} / 3$ trial in which UA was added during thrombolytic therapy administered to patients with acute cerebral infarction, Chamorro et al. [18] found that UA had a protective effect on the patient's nerve function. However, other studies have yielded different results. Sarfo et al. [19] found that UA concentration was positively correlated with the severity of cerebral infarction and positively correlated with early mortality in patients with acute cerebral infarction. Chen et al. [20] found that
TABLE 4: Univariate analysis for NHISS.

\begin{tabular}{lccc}
\hline Inflection point of UA & Effect size (OR) & $95 \%$ CI $P$ value & $P$ value \\
\hline$<372$ & 0.99 & 0.98 to 0.99 & $<0.0001$ \\
$\geq 372$ & 1.00 & 1.00 to 1.01 & 0.3487
\end{tabular}

Abbreviations: CI: confidence interval; OR: odds ratio.

serum UA level was not significantly correlated with the prognosis of patients with cerebral infarction. The wide variability in these reports can be attributed to different selected outcome indicators, research populations, and research types. Hence, comparisons among these findings are difficult.

The neuroprotective effects of UA can be explained in the following ways: (1) UA has an antioxidative effect, preventing the cerebrovascular system from being damaged by oxidative stress $[21,22]$. (2) UA can remove oxidative free radicals caused by chelation of metal ions and increase the metabolism of purine in the brain [23]. (3) UA protects local cerebral blood supply by activating the expression of neurotrophic factors and reduces brain damage caused by reperfusion $[24,25]$. It has been suggested in the literature that UA is an endogenous antioxidant and that low levels of UA are associated with the increased prevalence of neurodegenerative and inflammatory diseases of the central nervous system and deterioration of clinical processes. UA is rapidly consumed after acute cerebral infarction, and high levels of UA are associated with better prognoses [26]. This observation is consistent with our research conclusions.

Many previous studies have revealed a relationship between UA and the severity of cerebral infarction, and our study provides supporting evidence for the protective effect of UA on nerve function in patients with acute cerebral infarction. In this study, the nonlinear relationship between UA and the severity of cerebral infarction was determined, curve fitting was carried out, and the inflection point was calculated. The results were intuitive. It was found that a correlation remains, despite the adjustment of many confounding 
factors. A meta-analysis of 12739 patients with acute cerebral infarction suggested that, for patients with acute cerebral infarction, high levels of UA at onset have neuroprotective effects and are markers of a better prognosis, especially for patients undergoing thrombolytic therapy, regardless of the type of thrombolytic therapy [6]. This is basically consistent with the results of our study. It is necessary to consider whether patients with cerebral infarction treated with thrombolytic therapy could be given UA supplement, maintaining the upper limit of serum $\mathrm{UA}<372 \mu \mathrm{mol} / \mathrm{L}$, to minimize neurological damage. In particular, UA supplementation may have greater clinical significance in patients with acute episodes who cannot be treated with thrombolytic therapy. This will be our next research direction.

This study also has several limitations: (1) It was small, mainly comprising patients with acute primary cerebral infarction, and all participants were Chinese. (2) As a crosssectional study, it could not provide evidence of causality. Large prospective cohort studies are needed to investigate this. (3) Previous studies have shown that UA may be related to the size, type, and location of cerebral infarction [5, 27]. Studies have suggested that UA levels may be associated with secondary epilepsy following cerebral infarction [28], but these data were not collected in this study. (4) Patients with cerebral infarction may also have Parkinson's disease, decreased cognitive function, or other chronic diseases, which may also be related to UA levels. Due to the limitations of the original data, this avenue of research could not be further explored [29-31].

\section{Conclusion}

There was a nonlinear relationship between the UA level measured on admission and the degree of neurological impairment in patients with acute primary cerebral infarction. When UA was $<372 \mu \mathrm{mol} / \mathrm{L}$, it was negatively correlated with the degree of neurological impairment in patients with acute cerebral infarction, but when UA was $\geq 372 \mu \mathrm{mol} / \mathrm{L}$, the protective effect of UA disappeared.

\section{Data Availability}

The data sets used and/or analyzed in this study may be obtained by appropriate authorization or reasonable request.

\section{Conflicts of Interest}

The authors declare that they have no conflicts of interest.

\section{Authors' Contributions}

Ruying Wang and Yi Zhong have contributed equally to this work.

\section{Acknowledgments}

This study relies on the Clinical Medical Technology Innovation Guide Project (Approval No. 2017SK51308). The author is grateful to all the medical technicians in the Department of Neurology and Laboratory of the hospital. This work was supported by the Clinical Medical Technology Innovation Guidance Project [grant number 2017SK51308].

\section{References}

[1] D. E. D. San Gabriel and J. Slark, "The association of gout with an increased risk of hypertension and diabetes mellitus among stroke survivors in New Zealand: a cross-sectional study using routinely collected electronic health data," JRSM Cardiovascular Disease, vol. 8, 2019.

[2] The Lancet Neurology, "Adding stroke to the political agenda in Europe," The Lancet Neurology, vol. 18, no. 6, p. 513, 2019.

[3] Y. B. Chen, Z. Y. Zhou, G. M. Li et al., "Influences of an NR1I2 polymorphism on heterogeneous antiplatelet reactivity responses to clopidogrel and clinical outcomes in acute ischemic stroke patients," Acta Pharmacologica Sinica, vol. 40, no. 6, pp. 762-768, 2019.

[4] W. J. Tu, X. Dong, S. J. Zhao, D. G. Yang, and H. Chen, "Prognostic value of plasma neuroendocrine biomarkers in patients with acute ischaemic stroke," Journal of Neuroendocrinology, vol. 25, no. 9, pp. 771-778, 2013.

[5] N. Logallo, H. Naess, T. T. Idicula, J. Brogger, U. WajeAndreassen, and L. Thomassen, "Serum uri acid: neuroprotection in thrombolysis. The Bergen NORSTROKE study," BMC Neurology, vol. 11, no. 1, article 114, 2011.

[6] Z. Lei, J. Cai, H. Hong, and Y. Wang, "Serum uric acid level and outcome of patients with ischemic stroke: a systematic review and meta-analysis," The Neurologist, vol. 24, no. 4, pp. 121-131, 2019.

[7] N. Otani, S. Toyoda, M. Sakuma et al., "Effects of uric acid on vascular endothelial function from bedside to bench," Hypertension Research, vol. 41, no. 11, pp. 923-931, 2018.

[8] G. Di Stolfo, S. Mastroianno, D. R. Potenza et al., "Serum uric acid as a prognostic marker in the setting of advanced vascular disease: a prospective study in the elderly," Journal of Geriatric Cardiology, vol. 12, no. 5, pp. 515-520, 2015.

[9] Z. P. Li, L. B. Liang, C. Chen, S. Q. Li, and Z. M. An, "Association between serum uric acid and acute cerebral infarction: a case control study," Sichuan Da Xue Xue Bao. Yi Xue Ban, vol. 46, pp. 744-746, 2015.

[10] M. R. Heldner, P. Chaloulos-Iakovidis, L. Panos et al., "Outcome of patients with large vessel occlusion in the anterior circulation and low NIHSS score," Journal of Neurology, 2020.

[11] S. Majidi, M. Luby, J. K. Lynch et al., "MRI-based thrombolytic therapy in patients with acute ischemic stroke presenting with a low NIHSS," Neurology, vol. 93, no. 16, pp. e1507-e1513, 2019.

[12] V. Da Ros, J. Cortese, O. Chassin et al., "Thrombectomy or intravenous thrombolysis in patients with NIHSS of 5 or less?," Journal of Neuroradiology, vol. 46, no. 4, pp. 225-230, 2019.

[13] Y. Yang, Y. Zhang, Y. Li et al., "U-shaped relationship between functional outcome and serum uric acid in ischemic stroke," Cellular Physiology and Biochemistry, vol. 47, no. 6, pp. 2369-2379, 2018.

[14] A. Aliena-Valero, M. A. Lopez-Morales, M. C. Burguete et al., "Emergent uric acid treatment is synergistic with mechanical recanalization in improving stroke outcomes in male and female rats," Neuroscience, vol. 388, pp. 263-273, 2018.

[15] Y. Onetti, A. P. Dantas, B. Perez et al., "Middle cerebral artery remodeling following transient brain ischemia is linked to early postischemic hyperemia: a target of uric acid treatment," 
American Journal of Physiology. Heart and Circulatory Physiology, vol. 308, no. 8, pp. H862-H874, 2015.

[16] E. Chiquete, J. L. Ruiz-Sandoval, L. M. Murillo-Bonilla et al., "Serum uric acid and outcome after acute ischemic stroke: premier study," Cerebrovascular Diseases, vol. 35, no. 2, pp. 168174, 2013.

[17] J. C. Arevalo-Lorido, J. Carretero-Gomez, and N. R. Robles, "Serum uric acid levels and outcome during admission in acute ischaemic stroke, depending on renal function," The International Journal of Neuroscience, vol. 128, no. 10, pp. 906-912, 2018.

[18] A. Chamorro, S. Amaro, M. Castellanos et al., "Safety and efficacy of uric acid in patients with acute stroke (URICOICTUS): a randomised, double-blind phase 2b/3 trial," Lancet Neurology, vol. 13, no. 5, pp. 453-460, 2014.

[19] F. S. Sarfo, J. Akassi, N. K. Antwi et al., "Highly prevalent hyperuricaemia is associated with adverse clinical outcomes among Ghanaian stroke patients: an observational prospective study," Ghana Medical Journal, vol. 49, no. 3, pp. 165-172, 2015.

[20] L. H. Chen, C. Zhong, T. Xu et al., "Sex-specific association between uric acid and outcomes after acute ischemic stroke: a prospective study from CATIS trial," Scientific Reports, vol. 6, no. 1, article 38351, 2016.

[21] G. K. Glantzounis, E. Tsimoyiannis, A. Kappas, and D. Galaris, "Uric acid and oxidative stress," Current Pharmaceutical Design, vol. 11, no. 32, pp. 4145-4151, 2005.

[22] K. Oda, E. Kikuchi, E. Kuroda et al., "Uric acid, ferritin and $\gamma$ glutamyltransferase can be informative in prediction of the oxidative stress," Journal of Clinical Biochemistry and Nutrition, vol. 64, no. 2, pp. 124-128, 2019.

[23] H. Liu, G. P. Reynolds, W. Wang, and X. Wei, "Lower uric acid is associated with poor short-term outcome and a higher frequency of posterior arterial involvement in ischemic stroke," Neurological Sciences, vol. 39, no. 6, pp. 1117-1119, 2018.

[24] B. L. Ya, Q. Liu, H. F. Li et al., "Uric acid protects against focal cerebral ischemia/reperfusion-induced oxidative stress via activating Nrf2 and regulating neurotrophic factor expression," Oxidative Medicine and Cellular Longevity, vol. 2018, Article ID 6069150, 10 pages, 2018.

[25] B. Zhang, N. Yang, S. P. Lin, and F. Zhang, "Suitable concentrations of uric acid can reduce cell death in models of OGD and cerebral ischemia-reperfusion injury," Cellular and Molecular Neurobiology, vol. 37, no. 5, pp. 931-939, 2017.

[26] L. Llull, S. Amaro, and Á. Chamorro, "Administration of uric acid in the emergency treatment of acute ischemic stroke," Current Neurology and Neuroscience Reports, vol. 16, no. 1, article 4, 2016.

[27] S. Amaro, X. Urra, M. Gomez-Choco et al., "Uric acid levels are relevant in patients with stroke treated with thrombolysis," Stroke; a Journal of Cerebral Circulation, vol. 42, 1, Supplement 1, pp. S28-S32, 2010.

[28] D. Wang, B. Hu, Y. Dai et al., "Serum uric acid is highly associated with epilepsy secondary to cerebral infarction," Neurotoxicity Research, vol. 35, no. 1, pp. 63-70, 2019.

[29] T. D. Vannorsdall, A. M. Kueider, M. C. Carlson, and D. J. Schretlen, "Higher baseline serum uric acid is associated with poorer cognition but not rates of cognitive decline in women," Experimental Gerontology, vol. 60, pp. 136-139, 2014.
[30] A. Latourte, T. Bardin, and P. Richette, "Uric acid and cognitive decline: a double-edge sword?," Current Opinion in Rheumatology, vol. 30, no. 2, pp. 183-187, 2018.

[31] B. Vesely, E. Koritakova, N. I. Bohnen et al., "The contribution of cerebrovascular risk factors, metabolic and inflammatory changes to cognitive decline in Parkinson's disease: preliminary observations," Journal of Neural Transmission (Vienna), vol. 126, no. 10, pp. 1303-1312, 2019. 